\title{
Rings with a few more zero-divisors
}

\author{
C. Christensen
}

\begin{abstract}
It is well-known that every finite ring with non-zero-divisors has order not exceeding the square of the order $n$ of its left zero-divisor set. Unital rings whose order is precisely $n^{2}$ have been described already. Here we discuss finite rings with relatively larger zero-divisor sets, namely those of order greater than $n^{3 / 2}$. This is achieved by describing the class of all finite rings with left composition length two at most, and using a theorem relating the left composition length of a finite ring to the size of its left zero-divisor set.
\end{abstract}

It is known from the work of Ganesan ([2], [3]) and Koh [4] that a finite ring is either a field or its order is bounded above by $n^{2}$ where $n$ is the number of left zero-divisors of the ring. The class of unital rings where this bound is attained has been fully described by Corbas [1]; we refer to such rings as Corbas rings. In the present article we describe the class of all rings with left composition length at most two and show that it contains all rings of order greater than $n^{3 / 2}$. In fact, given a finite ring, we give a bound on its left composition length in terms of the size of its left zero-divisor set.

Throughout, $R$ denotes a finite ring and the set of left zero-divisors of $R$ is denoted by $\lambda R$. To avoid ambiguity: an element $r$ of $R$ is in $\lambda R$ if and only if $R$ has a non-zero element $s$ such that $r s=0$. As usual, given a finite set $X$, the symbol $|X|$ denotes the number of elements in $X$. Clearly, for any element $r$ of $R$, the property $|r S|<|S|$ for some subset $S$ of $R$ implies $r \in \lambda R$. [In 
particular therefore $\lambda R=\{r \mid r \in R \wedge r R \neq R\}=U\{I \mid I$ is a proper right ideal of $R$ \}.] Thus if $L / M$ is a composition factor of $R$ considered as an $R$-module in the natural way, then the annihilator of $L / M$ in $R$ is a subset of $\lambda R$. Let $x \in L-M$; then the kernel, ker $\theta$, of the $R$-module homomorphism $\theta: R \rightarrow L / M$ given by $\theta: r \leftrightarrow r x+M$ for all $r \in R$ annihilates $L / M$ and hence lies in $\lambda R$. It follows that $|R| \leq|L / M||\operatorname{ker} \theta| \leq|L / M||\lambda R|$ so that $|L / M| \geq|R||\lambda R|^{-1}$. From this one deduces inmediately:-

THEOREM 1. Let $|\lambda R| \leq|R|^{1-\varepsilon}$ where $\varepsilon$ is a positive real number less than 1 ; then every left composition factor of $R$ has order no less than $|R|^{\varepsilon}$ and hence the left composition length of $R$ is at most. $\frac{1}{\varepsilon}$.

In particular, therefore, the class of finite rings $R$ such that $|\lambda R|<|R|^{2 / 3}$ is contained in the class whose left composition length is at most two. We describe the latter class in the next theorem.

THEOREM 2. The left composition length of $R$ is at most two if and only if $R$ is one of the following types:-

(i) a fiezd;

(ii) a ring direct sum of two fietds;

(iii) a complete ring of $2 \times 2$ matrices over a field;

(iv) a ring direct sum of a field and a nulz ring of prime order;

(v) a ring direct sum of two nulz rings of prime orders;

(vi) a nulz ring with additive group of order $p$ or $p^{2}$ where $p$ is a prime;

(vii) $\left\langle a, b \mid p a=p b=0 \wedge a^{2}=b \wedge x^{3}=0\right\rangle$ where $p$ is a prime;

(viii) $\left(a \mid p^{2} a=0 \wedge a^{2}=p a\right)$ where $p$ is a prime;

(ix) $\left\{\left(\begin{array}{ll}a & b \\ 0 & 0\end{array}\right) \mid a, b \in K\right\}$ where $K$ is a fiezd;

(x) $\left\{\left(\begin{array}{ll}a & 0 \\ b & 0\end{array}\right) \mid a, b \in K\right\}$ where $K$ is a prime fiezd;

(xi) a Corbas ring. 
Proof. That all rings of types (i) to ( $x i$ ) have left composition length at most two is clear.

Conversely, let $R$ have left composition length at most two. Then if $R$ is semisimple it is of type (i), ( $i i)$ or ( $i i i)$. If $R$ is decomposable into a direct sum of two proper ideals, it is of type (ii), (iv) or (v). If $R$ is nilpotent and not directly decomposable then it is of type (vi) or $R^{2} \neq\{0\}$. For the latter case it is clear that $R>R^{2}>R^{3}=\{0\}$ is a left composition series for $R$ and it follows that the null rings $R^{2}$ and $R / R^{2}$ have no proper left ideals; consequently their additive groups are cyclic of prime order. Because $R$ is directly indecomposable it has prime power order and therefore $|R|=p^{2}$ for some prime $p$. If the additive group $R^{+}$of $R$ is elementary abelian then let $a$ and $c$ generate $R^{+}$ and choose $c \in R^{2}$. Then $a^{2}=k c$ for some positive integer $k$ where $p \nmid k$. Since $b=k c$ also generates $R^{2}, R$ is of type (vii). If, on the other hand, $R^{+}$is cyclic, let $b$ be one of its generators. Then $b$ has order $p^{2}$ and since $b^{2} \in R^{2}$ which is non-zero and has order $p$, it follows that $b^{2}=p k b$ for some positive integer $k$ coprime with $p$. Let $m$ be a positive integer such that $m k \equiv 1(\bmod p)$, then $a=m b$ also generates $R^{+}$and $a^{2}=p a$ so that $R$ is of type (viii).

It remains only to consider non-nilpotent, non-semisimple rings that are not directly decomposable. Let $J$ be the Jacobson radical of such a ring, then $R / J$ is a field and $J^{2}=\{0\}$ since $R$ has left composition length two. The condition $J^{2}=\{0\}$ allows us to define the multiplication $(p+J) j=r j$ for all $r+J \in R / J$ and $j \in J$. If $R$ is unital, then $J$ is a one-dimensional vector space over $R / J$ under this operation and hence $|J|=|R / J|$; it is now easy to check that $\lambda R=J$ so that $|R|=|\lambda R|^{2}$ and $R$ is a Corbas ring. Consider therefore the non-unital case. Let $e+J$ be the identity of $R / J$, then

$$
\left(3 e^{2}-2 e^{3}\right)^{2}-\left(3 e^{2}-2 e^{3}\right)=4\left(e^{2}-e\right)^{3}-3\left(e^{2}-e\right)^{2} \in J^{2}=\{0\}
$$

so that as usual $3 e^{2}-2 e^{3}$ is an idempotent congruent to $e \bmod J$. Without loss of generality assume that $e$ is itself idempotent. Since $e+J$ is the identity of $R / J$, the sets $\{r-r e \mid r \in R\}$ and $\{r$-er $\mid r \in R\}$ are both contained in $J$ and as we have assumed that $R$ is non-unital, at least one of them is not $\{0\}$. The first set is a left 
ideal and therefore is $\{0\}$ or $J$. The latter implies that $R=R e \oplus J$ and that $J e=\{0\}$. Moreover $R e$ is then a field $(\cong R / J)$ with identity $e$ in which case $J R=J R e=\{0\}$. Since by assumption $R$ is not directly decomposable as a ring, $R e$ is not an ideal so that $e J \neq\{0\}$ and as a left $R e$-module $J$ is a l-dimensional vector space. Therefore in this case $R$ is of type $(i x)$. It remains only to consider the case

$$
\{r-r e \mid r \in R\}=\{0\} \neq\{r-e r \mid r \in R\} .
$$

Thus $e$ is a right identity in $R$ and $\{r-e r \mid p \in R\}$ is a left ideal contained in and therefore equal to $J$. Hence $R=J \oplus e R, e R \cong R / J$ is a field and $J$ is unital as a right eR-module; moreover, as $R J=J^{2}+e R J=e J=\{0\}$ and $J$ is a minimal left ideal, $|J|$ must be a prime. It follows that $R$ is of type $(x)$.

It is a simple matter to deduce the following result from the preceding theorems.

COROLLARY. $|\lambda R|^{3 / 2}<|R|$ if and only if $R$ is of type (i), of type (ii) with the order $a, b$ of the fiezds satisfying $a^{2} b^{2}-(a+b-1)^{3}>0$, of type $(i x)$ or of type $(x i)$. The rings of types $(i x)$ and $(x i)$ are those where $|\lambda R|^{2}=|R|$.

\section{References}

[1] Basil Corbas, "Rings with few zero divisors", Math. Ann. 181 (1969), $1-7$.

[2] N. Ganesan, "Properties of rings with a finite number of zero divisors", Math. Ann. 157 (1964), 215-218.

[3] N. Ganesan, "Properties of rings with a finite number of zero divisors II", Math. Ann. 161 (1965), 24I-246.

[4] Kwangil Koh, "On 'Properties of rings with a finite number of zero divisors'", Math. Ann. 171 (1967), 79-80.

Department of Pure Mathematics, School of General Studies, Australian National University, Canberra, ACT. 